\title{
Uso potencial de la zeolita en la agricultura sustentable de la nueva revolución verde
}

\section{Potential use of zeolite on sustainable agriculture for the new green revolution}

\author{
Bulmaro Méndez-Argüello ${ }^{1}$, Ricardo Hugo Lira-Saldivar ${ }^{1 *}$ (๑) \\ ${ }^{1}$ Departamento de Biosistemas y Agrotecnología. Centro de Investigación en Química Aplicada, Blvd. Enrique Reyna No. 140, Col. San \\ José de los Cerritos. CP. 25294. Saltillo, Coahuila, México. \\ *Autor de correspondencia: hugo.lira@ciqa.edu.mx \\ Carta al editor recibida: 24 de abril de 2018 aceptada: 12 de diciembre de 2018
}

En los sistemas agrícolas emanados de la Revolución Verde, el empleo de agroquímicos sintéticos deteriora las condiciones del suelo, debido al incremento de metales pesados, agentes tóxicos y disminuye la fertilidad natural. Además, la poca o nula incorporación de materia orgánica que proporcionan los fertilizantes inorgánicos, hace que los microorganismos benéficos sean perjudicados y afecta la capacidad del suelo para retener agua y nutrientes (Solgi et al. 2018). La Nueva Revolución Verde implica una clara tendencia en promover una agricultura intensiva ecológica o sustentable, para hacer un uso eficiente de los insumos agrícolas sin deteriorar los agroecosistemas, lo que motiva la producción de alimentos con productos y tecnologías innovadoras (Martin-Guay et al. 2018). Para lograr mayor eficiencia en el uso de agroinsumos y el rendimiento de los cultivos, se requiere considerar que la absorción de nutrientes por las raíces se presenta en la zona de la rizósfera, lo que obliga a buscar nuevos productos que promuevan el crecimiento radicular y que se mantengan las condiciones óptimas para la absorción de agua y nutrientes para inducir mayor crecimiento vegetativo (Besset-Manzoni et al. 2018).

Materiales naturales como las rocas zeolíticas no presentan los problemas y desventajas de los fertilizantes convencionales, por lo que su empleo para promover una agricultura sustentable es conveniente, principalmente en países que tienen yacimientos de estos minerales, como el caso de México (Ostrooumov et al. 2012). Las zeolitas son fáciles de producir en términos de costos energéticos y de beneficio para mejorar la estructura y disponibilidad de nutrientes del suelo, así como para promover la producción ecológica de alimentos. Las zeolitas son aluminosilicatos porosos de origen volcánico que poseen alta capacidad de intercambio catiónico y gran afinidad por iones amonio $\left(\mathrm{NH}_{4}^{+}\right)$; además contienen cantidades considerables de nutrientes como $\mathrm{K}, \mathrm{Mg}$, Ca y Na (Auerbach et al. 2003). También beneficios notorios debido a que los nutrientes del suelo o de las soluciones nutritivas aplicadas, son retenidas en su estructura tipo panal y luego son liberados de forma lenta (Bybordi et al. 2014). La mezcla de zeolita con fertilizantes nitrogenados ayuda a reducir la lixiviación de los nitratos, mejorando la calidad del suelo y haciendo disponibles los nutrientes por más tiempo, mientras que en suelos de textura arenosa y con alta disponibilidad hídrica, reducen los niveles de $\mathrm{NO}_{3}$ lixiviables, sin afectar el crecimiento de las plantas debido a la retención de $\mathrm{N}$ en su estructura porosa (Leyva-Ramos et al. 2004). Investigaciones recientes señalan que la zeolita tiene beneficios en el crecimiento $y$ rendimiento de algunos cultivos a cielo abierto. Al respecto Lira-Saldivar et al. (2017) reportan que este mineral mejora de forma significativa la producción de Cucurbita pepo con y sin acolchado plástico (AP), presentando las plantas de calabacita tratadas con zeolita y AP mayor tasa fotosintética durante todo el día; pero también la zeolita sola mostró resultados positivos, ya que la incorporación de $40 \mathrm{t} \mathrm{ha}^{-1}$ al suelo, incrementó el número de flores, biomasa seca, calidad y rendimiento de frutos. También se ha señalado que la adición de zeolita al suelo permite aplicar menores cantidades de fertilizantes y favorece 
el uso eficiente del agua, debido a que retiene y libera gradualmente el agua y los nutrientes (Tsintskaladze et al. 2017).

La zeolita también puede ser utilizada para mejorar la calidad de los suelos agrícolas, ya que, debido a su microestructura en forma de panal, puede absorber plaguicidas, lo que la hace eficaz en la protección de cultivos contra plagas y enfermedades (De Smedt et al. 2015). Estas propiedades también permiten que las zeolitas puedan actuar como vehículos transportadores de diferentes sustancias activas, siendo posible el uso de zeolitas para la aplicación gradual de productos biológicos y químicos en la agricultura (Zahedi et al. 2009). Debido a lo antes señalado, la zeolita tiene potencial en la agricultura sustentable, ya que permite incrementar la eficiencia de los agroinsumos, mejorar la estructura del suelo y proteger a los cultivos. En México se tienen grandes reservas de este mineral en los estados de Oaxaca, Sonora, Puebla, San Luis Potosí, Michoacán y otras entidades, por lo que es una alternativa ecológica y factible para la agricultura de riego y temporal. Además, su costo es relativamente bajo para incorporarla al suelo y recibir sus beneficios por varios años, lo que resulta conveniente para mejorar la relación costo: beneficio de los productores agropecuarios.

\section{LITERATURA CITADA}

Auerbach SM, Carrado K, Dutta PK (2003) Handbook of zeolite science and technology. CRC press. New York, USA.

Besset-Manzoni Y, Rieusset L, Joly P, Comte G, Prigent-Combaret C (2018) Exploiting rhizosphere microbial cooperation for developing sustainable agriculture strategies. Environmental Science and Pollution Research 1: $1-18$.

Bybordi A, Saadat S, Zargaripour P (2017) The effect of zeolite, selenium and silicon on qualitative and quantitative traits of onion grown under salinity conditions. Archives of Agronomy and Soil Science 1: 1-11.

De Smedt C, Someus E, Spanoghe P (2015) Potential and actual uses of zeolites in crop protection. Pest Management Science 71: 1355-1367.

Leyva-Ramos R, Aguilar-Armenta G, Gonzalez-Gutierrez LV, Guerrero-Coronado RM, Mendoza-Barron J (2004) Ammonia exchange on clinoptilolite from mineral deposits located in Mexico. Journal of Chemical Technology \& Biotechnology: International Research in Process, Environmental \& Clean Technology 79: 651-657.

Lira-Saldivar RH, Méndez-Argüello B, Felipe-Victoriano M, Vera-Reyes I, Cárdenas-Flores A, Ibarra-Jiménez L (2017) Gas exchange, yield and fruit quality of Cucurbita pepo cultivated with zeolite and plastic mulch. Agrochimica 61: 1-17.

Martin-Guay, MO, Paquette A, Dupras J, Rivest D (2018) The New Green Revolution: Sustainable intensification of agriculture by intercropping. Science of the Total Environment 615: 767-772.

Ostrooumov M, Cappelletti P, de-Gennaro R (2012) Mineralogical study of zeolite from New Mexican deposits (Cuitzeo area, Michoacan, Mexico). Applied Clay Science 55: 27-35.

Solgi E, Sheikhzadeh H, Solgi M (2018) Role of irrigation water, inorganic and organic fertilizers in soil and crop contamination by potentially hazardous elements in intensive farming systems: Case study from Moghan agro-industry, Iran. Journal of Geochemical Exploration 185: 74-80.

Tsintskaladze G, Eprikashvili L, Mumladze N, Gabunia V, Sharashenidze T, Zautashvili M, Shatakishvili T (2017) Nitrogenous zeolite nanomaterial and the possibility of its application in agriculture. Annals of Agrarian Science 15: 365-369. 
Zahedi H, Noormohammadi G, Rad AS, Habibi D, Boojar MMA (2009) The effects of zeolite and foliar applications of selenium on growth, yield and yield components of three canola cultivars under drought stress. World Applied Sciences Journal, 7: 255-262. 
